\title{
Ein Glossar für die strukturierte Befundung radiologischer Untersuchungen des Thorax
}

\author{
German Glossary for Structured Reporting in Chest Radiology
}

S. Diederich

Institut

Diagnostische und Interventionelle Radiologie, Marien Hospital Düsseldorf, Germany

\section{Bibliografie}

Dol http://dx.doi.org/10.1055/ s-0035-1553222

Fortschr Röntgenstr 2015; 187: 637 (c) Georg Thieme Verlag KG Stuttgart - New York ·

ISSN 1438-9029

\section{Korrespondenzadresse}

Prof. Dr. med. Stefan Diederich

Diagnostische und Interventio-

nelle Radiologie, Marien Hospital

Düsseldorf

Rochusstr. 2

40479 Düsseldorf

Germany

Tel.: ++ 49/211/44002101

Fax: ++49/211/44002102

stefan.diederich@vkkd-

kliniken.de
In der aktuellen Ausgabe der RöFo erscheint das „Glossar thoraxradiologischer Begriffe entsprechend der Terminologie der Fleischner Society“ von Wormanns et al. als deutsche Version der entsprechenden englischsprachigen Publikation der Fleischner Society, der internationalen und interdisziplinären Autorität in der Medizin und insbesondere der Bildgebung des Thorax.

Ein Glossar ist eine Liste von Wörtern mit beigefügten Erklärungen oder Übersetzungen (Wikipedia). Dieses Glossar listet die in der Befundung thoraxradiologischer Untersuchungen gebräuchlichen und sinnvollen Begriffe auf, liefert präzise Beschreibungen und Definitionen und illustriert sie mit Bildbeispielen.

Die englischsprachige Vorlage ist „die“ Grundlage kompetenter Befundung thoraxradiologischer Untersuchungen. Die Publikation von Wormanns et al. stellt nicht nur eine sehr gute Übersetzung des Originals dar, sondern übertrifft dieses teilweise noch in der Qualität der Illustrationen. Darüber hinaus berücksichtigt sie die praktische Anwendung in Deutschland.

Ähnlich wie die Vorlage im angloamerikanischen Sprachraum sollte dieses Glossar die Grundlage jedes Befundes in der projektionsradiografischen Röntgendiagnostik und Computertomografie des Thorax werden.

\section{Warum sollte ich mich als Radiologe an dem Glossar orientieren? $\nabla$}

Traditionell ist die Beschreibung radiologischer Untersuchungen, insbesondere im deutschsprachigen Raum, sehr von individuellen Vorlieben und Erfahrungen des Befunders geprägt. Dabei wurde lange eine möglichst vielfältige Sprache zur erhofften Differenzierung in der Beschreibung verwendet, gerne auch durch Vergleiche mit Strukturen aus anderen zentralen Lebensbereichen („hirsekorngroß“, „haselnussgroß“, „,wallnussgroß“, „Kindskopfgroß“ etc.). Viele Begriffe sorgten beim Leser des schriftlichen Befundes eher für vage Assoziationen als für präzise Vorstellungen („,wolkige, flaue Verdichtung der Lunge“). Im Ergebnis führte dies zu sehr unterschiedlichen schriftlichen Befunden für identische Phänomene und verhinderte die teils gut möglichen Rückschlüsse vom radiologischen Befund auf dessen pathomorphologisches Substrat.

Die Verwendung eines verbindlichen, präzise definierten Vokabulars sorgt dagegen für eine Vereinheitlichung in der Beschreibung des gleichen bildgebenden Befundes durch unterschiedliche Befunder und führt zumindest teilweise direkt zum makroanatomischen Korrelat. Damit wird unmittelbar die Differenzialdiagnose des Befundes präzisiert.

Der Befunder selbst wird zwangsläufig zu einer möglichst präzisen Analyse der Befunde gezwungen - präziser als dies die unstrukturierte Verwendung undefinierter Begriffe erfordern würde. Zwar ergibt sich eine Einschränkung der persönlichen sprachlich-gestalterischen Freiheiten, wobei es allerdings nahe liegt, diese eher durch die Verfassung von Gedichten oder Essays als durch radiologische Befunde auszuleben.

Im Ergebnis wird die Verwendung eines und gerade auch diesen Glossars dazu führen, dass radiologische Befunde präziser, vergleichbarer und damit für die Patientenversorgung relevanter werden. Sie kann dazu führen, den Stellenwert der Radiologie bei den überweisenden Disziplinen zu steigern und vor allem stellt sie einen Beitrag zur möglichst guten Versorgung unserer Patienten dar.

Insofern ist zu wünschen, dass möglichst jeder deutschsprachige Radiologe dieses Glossar für jede Befundung einer Thoraxuntersuchung verwendet. 